\title{
Factors influencing diagnostic accuracy of endoscopic ultrasound-guided fine-needle aspiration (EUS-FNA) in pancreatic and biliary tumors
}

Giovanna Del Vecchio Blanco, Giampiero Palmieri, Diana Giannarelli, Vincenzo Formica, Ilaria Portarena, Antonella Nardecchia, Edoardo Troncone, Michela Benassi, Emilia Giudice, Alessandro Anselmo, Giuseppe Tisone, Mario Roselli, Giovanni Monteleone \& Omero Alessandro Paoluzi

To cite this article: Giovanna Del Vecchio Blanco, Giampiero Palmieri, Diana Giannarelli, Vincenzo Formica, Ilaria Portarena, Antonella Nardecchia, Edoardo Troncone, Michela Benassi, Emilia Giudice, Alessandro Anselmo, Giuseppe Tisone, Mario Roselli, Giovanni Monteleone \& Omero Alessandro Paoluzi (2021) Factors influencing diagnostic accuracy of endoscopic ultrasound-guided fine-needle aspiration (EUS-FNA) in pancreatic and biliary tumors, Scandinavian Journal of Gastroenterology, 56:4, 498-504, DOI: 10.1080/00365521.2021.1880628

To link to this article: https://doi.org/10.1080/00365521.2021.1880628

Published online: 04 Feb 2021.

Џ Article views: 49
Submit your article to this journal $\pi$

\section{View Crossmark data $₫$}




\title{
Factors influencing diagnostic accuracy of endoscopic ultrasound-guided fine-needle aspiration (EUS-FNA) in pancreatic and biliary tumors
}

\author{
Giovanna Del Vecchio Blanco ${ }^{a}$, Giampiero Palmieri ${ }^{b}$, Diana Giannarellic, Vincenzo Formica ${ }^{\mathrm{d}}$, llaria Portarena ${ }^{\mathrm{d}}$, \\ Antonella Nardecchia ${ }^{d}$, Edoardo Troncone ${ }^{a}$, Michela Benassi ${ }^{e}$, Emilia Giudice ${ }^{e}$, Alessandro Anselmo ${ }^{f}$, \\ Giuseppe Tisone ${ }^{\mathrm{f}}$, Mario Roselli ${ }^{\mathrm{d}}$, Giovanni Monteleone ${ }^{\mathrm{a}}$ and Omero Alessandro Paoluzi ${ }^{\mathrm{a}}$ \\ ${ }^{a}$ Gastroenterology Unit, Department of Systems Medicine, University of Rome Tor Vergata, Rome, Italy; ${ }^{b}$ Anatomic Pathology Unit, \\ Department of Biomedicine and Prevention, University of Rome Tor Vergata, Rome, Italy; 'Biostatistical Unit, Regina Elena Institute-Hospital

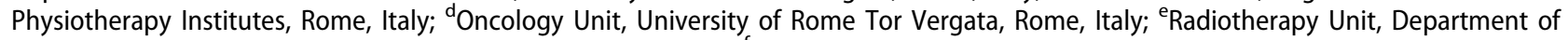 \\ Oncohematology, University of Rome Tor Vergata, Rome, Italy; ${ }^{\mathrm{f}}$ Transplant Surgery Unit, Department of Surgery, University of Rome Tor \\ Vergata, Rome, Italy
}

\begin{abstract}
Background and aim: Diagnostic accuracy of endoscopic ultrasound-guided fine-needle aspiration (EUS-FNA) is influenced by several factors, primarily operator expertise. Formal training in EUS-FNA, as suggested by the European Society of Gastrointestinal Endoscopy and the American Society for Gastrointestinal Endoscopy guidelines, is not always available and is often expensive and time-consuming. In this study we evaluate factors influencing the diagnostic accuracy of pancreatic EUS-FNA.

Methods: In a retrospective study, 557 consecutive EUS-FNAs were evaluated. Several variables relating to the procedures were considered to calculate the EUS-FNA performance over eight years.

Results: A total of 308 out of 557 EUS-FNAs were selected. Overall sensitivity of EUS-FNA was $66 \%$ (95\% Cl: 60.8-71.8), specificity 100\%, and diagnostic accuracy $69 \%$ (95\% Cl: 64.0-74.4). An increase in diagnostic accuracy was observed to $>90 \%$ using a new fine-needle biopsy (FNB) needle and in the case of simultaneous sampling of primary and metastatic lesions. Diagnostic accuracy $>80 \%$ was observed after 250 procedures, in the absence of rapid on-site cytopathological examination (ROSE). Multivariate logistic regression analysis confirmed that the FNB needle, operator skill, and double EUSFNA sampling are associated with high diagnostic accuracy.

Conclusions: The learning curve for EUS-FNA may be longer and a considerable number of procedures are needed to achieve high diagnostic accuracy in the absence of ROSE. However, the use of FNB needles and the simultaneous sampling of primary and metastatic lesions can rapidly improve the diagnostic accuracy of the procedure.
\end{abstract}

\section{ARTICLE HISTORY}

Received 16 December 2020

Revised 16 January 2021

Accepted 19 January 2021

\section{KEYWORDS}

Pancreatic cancer; endoscopic ultrasonography; EUS-guided fineneedle aspiration

\section{Introduction}

Endoscopic ultrasound (EUS) is a diagnostic technique combining endoscopic viewing and ultrasonography imaging. EUS is used worldwide for diagnosing and staging several kinds of gastrointestinal, mediastinal, and perirectal space malignancies $[1,2]$ as it allows a detailed analysis of the parietal wall of the digestive tract and surrounding organs. The major advantage of this technique is the ability to collect tissue from a lesion suspected to be malignant by EUS-guided fine-needle aspiration (EUS-FNA). EUS-FNA has proven to be effective for the diagnosis of several types of primary or metastatic tumors [3-6], biliopancreatic and abdominal/mediastinal lymph nodes mass lesions $[7,8]$. EUS-FNA is generally safe; rare complications after this procedure can be self-limited bleeding from the needle insertion site, pancreatitis, and infection, which account for $0-2 \%$ of cases $[9,10]$. In patients with pancreatic mass, EUS-FNA is considered the method of choice for differential tissue diagnosis between a benign or malignant lesion, yielding extremely high sensitivity (85\%-89\%) and specificity (96\%-99\%) [2]. EUS-FNA is also helpful in choosing the most appropriate targeted therapy in a locally advanced or borderline resectable neoplasm on the pancreas and is often requested by the surgeon to confirm the diagnosis of malignancy before surgery.

Multiple factors influence the diagnostic accuracy of EUSFNA in the differential diagnosis of pancreatic neoplasms, above all the size and location of the mass, and the presence of peritumoral desmoplastic stromal reaction or concomitant chronic pancreatitis. Some methodological innovations have been suggested to improve the diagnostic yield of EUS-FNA, such as increasing the needle gauge, modifying the needle tip, increasing the number of passes in the lesion, or modifying the sampling technique (standard versus stylet slow-pull suction, wet method, fanning method) [6,11-14].

There is general agreement that the diagnostic accuracy of EUS-FNA increases with operator experience, as it is an operator-dependent technique with a long learning curve 
[11-21]. Based on expert opinion, the American Society for Gastrointestinal Endoscopy (ASGE) suggests that clinical competence in all aspects of EUS may be achieved following at least 150 supervised EUS procedures. As a part of formal training, at least 75 supervised EUS procedures with pancreatic-biliary indication and 60 FNAs, including 25 of the pancreas, should be performed during the learning period [20]. Guidelines released by the European Society of Gastrointestinal Endoscopy (ESGE) recommend a minimum of 20 to 30 supervised EUS-FNAs in the presence of rapid onsite cytopathological examination (ROSE) to reach sufficient competency in this technique and sensitivity for pathological diagnosis up to $80 \%$ [21]. The importance of ROSE in increasing EUS-FNA diagnostic accuracy and reducing the number of passes needed to obtain sufficient tissue specimens is largely debated [22]. However, the presence of a cytopathologist in the endoscopy room may be limited for a number of practical reasons, such as local availability and high costs.

The present study aimed to evaluate EUS-FNA performance in the diagnosis of pancreatic and biliary neoplasms and to identify factors influencing diagnostic accuracy in the absence of ROSE.

\section{Patients and methods}

\section{Design of the study and population}

From January 2010 to May 2018, all clinical data regarding patients undergoing EUS-FNA for suspected biliary or pancreatic neoplasia in our Gastroenterology Unit were recorded in an electronic database at the time of the procedure. Data included were demographic information, indication for EUSFNA, findings of previous diagnostic tests, and any active symptoms. The type and size of needle used, the sampling technique (standard versus stylet slow-pull suction), the number of passes, and the site of the lesion were also recorded. The final diagnosis was defined based on surgical specimens or the outcome at follow-up. All procedures were part of a standard diagnostic work-up and were performed after written informed consent was given. A multidisciplinary team established the diagnostic and therapeutic work-up of each patient. Patients with a final diagnosis different from pancreatic or biliary carcinoma, incomplete data, or an inconclusive diagnosis at the end of the diagnostic work-up were excluded from the study analysis.

\section{EUS-FNA technique}

All procedures were performed by a single operator (DVB.G.), a gastroenterologist skilled in digestive endoscopy, who had undergone a training period in a center with a high volume of EUS procedures. During the traineeship, 200 EUS procedures including EUS-FNA were observed. Having carried out at least 250 diagnostic EUS procedures and acquired a good level of competence in linear EUS, the operator started performing EUS-FNA without supervisor. Subsequently, she attended further live EUS courses and international EUS meetings to gain further experience in interventional EUS.
All EUS-FNAs were performed with the patient recumbent in the left lateral position, under intravenous conscious or deep sedation with midazolam plus fentanyl or propofol. Vital signs, including heart rate, blood pressure, and partial pressure of oxygen $\left(\mathrm{paO}_{2}\right)$ in the blood, were continuously monitored during the procedure and in the three hours following. Outpatients were discharged when a normal consciousness state and physical ability were fully recovered. A linear array echoendoscope equipped with a $7.5 \mathrm{MHz}$ transducer (GF-UCT140 or GF-UCT 180 Olympus, Tokyo, Japan) was used for diagnosing and staging the lesions. EUS-FNAs were carried out using a 19-, 22-, or 20-gauge needle (EchoTip ProCore ${ }^{\circledR}$, Wilson-Cook Medical Inc, Winston-Salem, NC, USA; Expect ${ }^{\mathrm{TM}}$ or Acquire $^{\mathrm{TM}}$, Boston Scientific, Marlborough, MA, USA: Shark Core ${ }^{\mathrm{TM}}$ FNB Medtronic, Minneapolis, MN, USA) for trans-duodenal and trans-gastric sampling. The needle was inserted through the working channel of the echoendoscope and advanced into the target lesion under real-time EUS imaging. During the study period, the suction technique changed from standard, with a $10-\mathrm{mL}$ syringe attached to the proximal end of the needle, to the slow-pull technique. The needle was moved back and forth within the lesion for a minimum of two to a maximum of five times. If the pathologist returned a negative or inconclusive result due to inadequate sampling, and in the event of high clinical suspicion of malignancy, EUS-FNA was repeated at least two times. In patients with metastasis, EUS-FNA included primary and secondary lesions in the same session, performing tissue sampling first in the metastasis and then in the primary lesion.

Tissue specimens were immediately put into formalin by releasing the syringe in a specimen bottle and adequacy of the material was evaluated in all patients by gross inspection by the operator, as ROSE was not available. Any complication during or following the procedure was recorded.

In all patients, the final diagnosis of malignancy was determined on the pathological findings of surgery specimens or clinical outcomes (i.e. death from the disease, radiological evidence by CT scan, or MRI of disease progression). The follow-up period was 252 days (range: 33-1355). Patients with no neoplastic findings on EUS-FNA were followed up for at least one1 year. EUS-FNAs with a conclusive diagnosis of malignancy were considered as true positive while those with no evidence of neoplasia or nuclear atypia as true negative samples. To evaluate the influence of the operator's skills on the diagnostic accuracy of EUS-FNA, all procedures carried out during the study period were stratified over time in blocks of 50 (1-50, 51-100, 101-150, 151-200, 201-250, >250).

\section{Histological evaluation}

Specimens were embedded in paraffin and sections stained with hematoxylin-eosin. Information regarding clinical history, including a description of the target lesion and the results of relevant diagnostic tests, was sent to the pathologist at the time of tissue sample submission. 


\section{Statistical analysis}

Performance of EUS-FNA was evaluated according to the location of the lesion (pancreatic head, body, tail), needle size (19-, 20-, 22-gauge), number of passes $(1-2,3-4,5-6)$, needle type, site of the lesion (primary and metastatic lesion), and operator skill (based on the number of procedures performed). All these factors were considered as variables potentially influencing diagnostic accuracy and their weight was evaluated by a logistic regression model. Variables were selected using a stepwise forward method based on likelihood ratio and remove and enter limits were set to 0.075 and 0.10 , respectively, to better describe the possible associations. All statistical analyses were calculated using IBM SPSS ver. 21.0 statistical software.

\section{Results}

During the study period, 308 out of 557 EUS-FNAs were performed in 283 consecutive patients, 151 males, and 132 females, with a mean age of 66 years (range: 23-87), the remaining 249 procedures were excluded from the analysis as performed for indications other than biliopancreatic diseases. The final diagnosis was solid pancreatic neoplasm in 253 patients, cystic pancreatic neoplasm in eight patients, liver primary or metastatic neoplasm of previously diagnosed tumors in five patients, and cholangiocarcinoma in 17 patients.

Based on histological findings, the final pancreatic tissue diagnosis was pancreatic neuroendocrine tumor in 19 patients, pancreatic signet ring cell carcinoma in nine patients, poorly differentiated pancreatic carcinoma in 142 patients, B-cell lymphoma in two patients, pancreatic metastasis from previous kidney carcinoma in four patients, pancreatic metastasis from prostate carcinoma in one patient, and previous lung cancer in one patient.

Forty-eight EUS-FNAs were carried out using a 19-gauge needle. A total of 223 EUS-FNAs were carried out using a 22gauge needle; 42 of these 223 procedures were performed using a new fine-needle biopsy (FNB) needle during the final year of the study. EUS-FNAs were performed using a 25-gauge needle in five cases and 22- plus 25-gauge needles at the same time in the remaining cases. The number of passes in each lesion was initially $4-5$ (30\% of cases) and decreased to three during the last three years of the study period.

At the end of the analysis, 188 samples were categorized as true positive (60\%), 26 samples as true negative (9\%), and 94 samples as not diagnostic (31\%), with an overall EUS-FNA sensitivity, specificity, and diagnostic accuracy of $66.3 \%(95 \%$ $\mathrm{Cl}$ : 60.8-71.8), $100 \%$, and $69.2 \% \quad(95 \% \mathrm{Cl}: \quad 64.0-74.4)$, respectively.

The simultaneous sampling of primary and metastatic lesions and the repetition of EUS-FNA two times were found to increase diagnostic accuracy up to $97.9 \% \quad(95 \% \mathrm{Cl}$ : 93.9-100) and $98 \%$ (95\% Cl: 93.9-100), respectively (Figure 1).

By stratifying data according to the site of the lesion, diagnostic accuracy was $72.3 \%$ in a neoplasm in the pancreatic head versus $75.2 \%$ in the pancreatic body and tail, and in other sites.

The majority of EUS-FNAs were performed with a 22gauge needle with an overall sensitivity and diagnostic accuracy of $57.7 \%(95 \% \mathrm{Cl}: 50.1-65.2)$ and $61.7 \%(95 \% \mathrm{Cl}$ : 54.6-68.8), respectively. An improvement in EUS-FNA performance (sensitivity: $72.4 \%$ [95\% Cl: 62.3-82.4], diagnostic accuracy: $74.7 \%$ [95\% Cl: 65.3-84.1]) was obtained by changing the needle during the procedure from 22- to 25-gauge (Figure 1) in pancreatic head neoplasms. A considerable increase in sensitivity and diagnostic accuracy, up to $~ 90.5 \%$ (95\% Cl: 80.7-99.3), was observed in the case of EUS-FNB sampling (Figure 2).

To evaluate the influence of the operator's skill on the performance of EUS-FNA, sensitivity and diagnostic accuracy were calculated by stratifying the procedures over time into six blocks of 50 (Table 1). In the absence of ROSE, sensitivity and diagnostic accuracy progressively increased according to operator experience, with values constantly $>80 \%$ after the first 250 procedures.

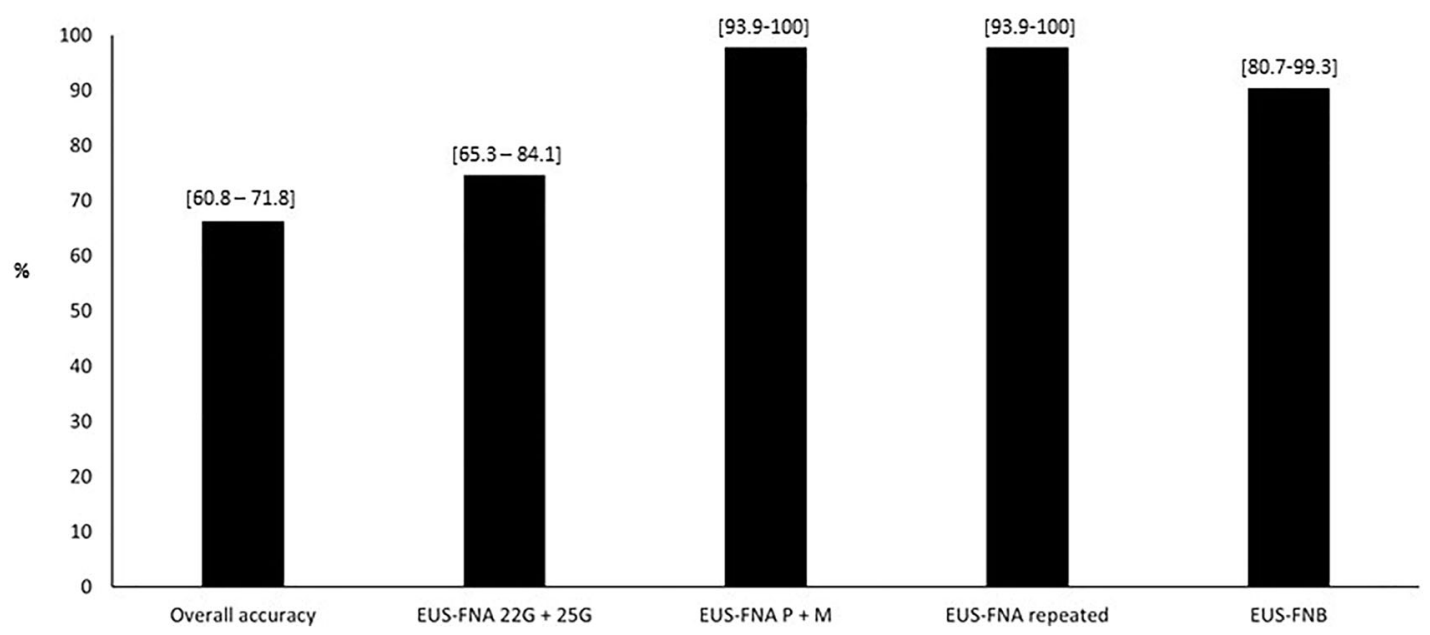

Figure 1. Diagnostic accuracy of EUS-FNA according to subgroup considered: 12 cases performed with a change in needle during the procedure from 22 - to 25 gauge, 33 cases with double simultaneous sampling in primary (P) and metastatic (M) lesions, 24 cases repeating EUS-FNA two times, and 42 cases using EUS-FNB. Number in brackets are $95 \%$ IC. 


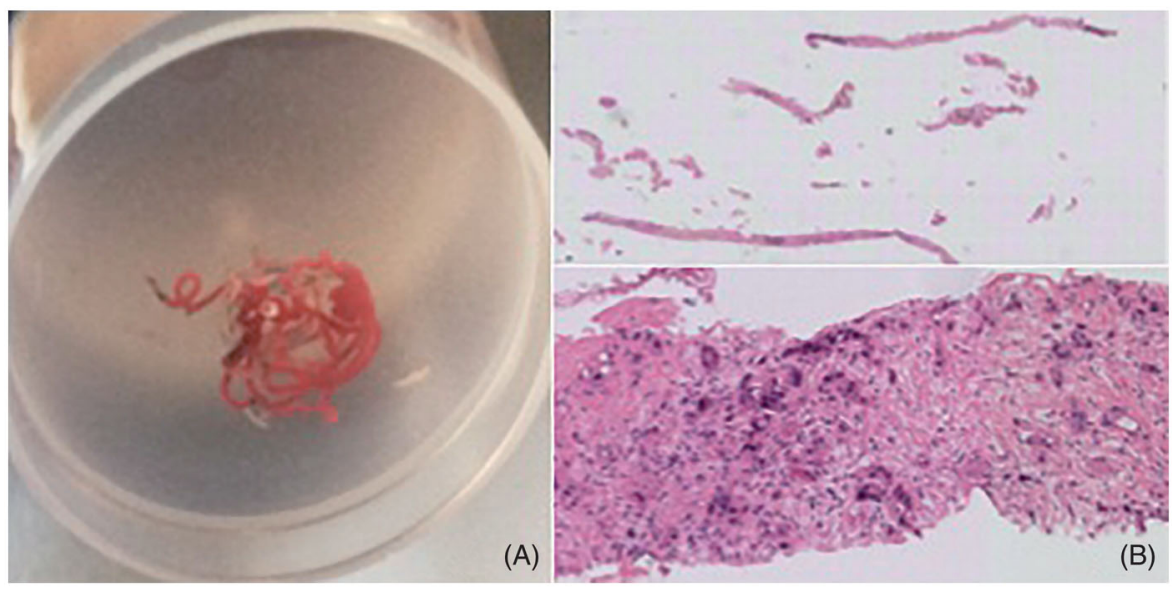

Figure 2. (A) EUS-FNB samples fixed in formalin. (B) Core biopsy of solid pancreatic neoplasia stained with hematoxylin-eosin at two different magnifications.

Table 1. Sensitivity and diagnostic accuracy in six groups of 50 procedures consecutively carried out during the study period.

\begin{tabular}{lcc}
\hline Number of cases & Sensitivity $(95 \% \mathrm{Cl})$ & Accuracy $(95 \% \mathrm{Cl})$ \\
\hline $1-50$ & $52.3(37.5-67.0)$ & $58.0(44.3-71.7)$ \\
$51-100$ & $53.2(38.9-67.5)$ & $56.0(42.2-69.8)$ \\
$101-150$ & $59.6(45.5-73.6)$ & $62.0(48.6-75.5)$ \\
$151-200$ & $73.2(59.6-86.7)$ & $78.0(66.5-89.5)$ \\
$201-250$ & $69.6(56.3-82.9)$ & $71.4(58.8-84.1)$ \\
$>251$ & $87.0(78.1-96.0)$ & $87.5(78.8-96.2)$ \\
\hline
\end{tabular}

Logistic regression analysis confirmed that the variables associated with diagnostic accuracy were the endosonographer's ability (OR $=1.21,95 \% \mathrm{Cl}: 1.02-1.43, p=.03)$ and the simultaneous sampling of primary and metastatic lesions (OR $=9.67,95 \% \mathrm{Cl}: 1.27-73.75], p=.03)$. An association was found between diagnostic accuracy and the use of EUS-FNB needle type, even if it did not reach statistical significance $(\mathrm{OR}=3.06,95 \% \mathrm{Cl}: 0.94-9.9, p=.06)$.

\section{Discussion}

EUS with FNA or FNB is currently the method of choice for tissue acquisition and is useful to differentiate between malignant and benign lesions in pancreas and biliary tract. When performed by an expert operator, interventional EUS has high diagnostic accuracy and a low rate of complications $[9,10,21,23]$. To achieve competency, a supervised training period is recommended by ESGE and ASGE guidelines, which suggest performing from 20-30 to 60 supervised EUS-FNAs to reach sufficient expertise in interventional EUS $[20,21,24-26]$. However, information in the literature is limited as to the best way to train future endosonographers. The exact number of procedures needed to achieve competence in EUS-FNA is difficult to establish and has not yet been sufficiently validated. Adequate EUS training is complex, expensive, and requires a long period of learning, which is not always available to an experienced operator working full time in a hospital setting. Furthermore, adequate knowledge of the pancreatic biliary anatomy and of specific diseases involving these organs [10,19-21] is required before starting EUS-FNA. In some instances, EUS training programs do not fulfill ASGE recommendations, as reported in a survey by Azad et al. [27]. The survey stated that only $48 \%$ of advanced fellows performed the minimum number of EUS procedures recommended by the ASGE. Only just over $50 \%$ of training centers had an annual EUS caseload of at least 200 procedures, which translates into the possibility to train one trainee each year [28]. Therefore, although mentored training is the best way to achieve competency in EUS-FNA, it is conceivable that a substantial proportion of endosonographers do not undergo a training program and their expertise grows through self-training. This may strongly influence the learning process in EUS-FNA techniques, signifying a long learning curve and a greater number of procedures before adequate proficiency is acquired. The impact of the operator's ability on the diagnostic accuracy of EUS-FNA was confirmed by several investigations. Harewood et al. [17] reported that the experience of the operator is the only variable affecting the diagnostic accuracy of EUS-FNA, whilst the location and size of the lesion do not seem to influence the cellularity of the sample and pathology results. The expertise of the endosonographer is reported to affect the number of EUS-FNA passes and the safety of the procedure. In a prospective study evaluating 300 procedures performed by a single operator, the percentage of EUS-FNA with $>5$ passes decreased after 100 procedures and the number of complications after 200 [19]. The number of passes during EUS-FNA procedures was found to be the main factor influencing diagnostic accuracy in a series of EUS-FNAs of pancreatic and peripancreatic mass carried out without an on-site cytopathologist [29].

In the present study, all the procedures were performed by a single operator who had undergone training in diagnostic EUS, not including supervised EUS-FNAs. The sensitivity and diagnostic accuracy of EUS-FNA improved after the first 50 cases but reached a value $>80 \%$ after 150 procedures. In this investigation, the number of procedures required to reach good diagnostic accuracy during the learning curve was greater than that reported in previous studies [17,19,21]. This discrepancy may be explained by the absence of ROSE and by the relatively limited experience of the pathologist in evaluating pancreatic FNA at the beginning of the study period. The need for a pathologist's training must be taken into consideration in initiating the EUS FNA procedure, since 
the learning curve involves both the endosonographer and the pathologist.

To overcome these limitations, as recommended by ESGE guidelines [6,21], during the same procedure a double sampling was performed, when possible, of primary and suspected metastatic lesions such as in liver or lymph nodes. Otherwise, EUS-FNA was repeated two times in the case of an inconclusive diagnosis. This working modality increased diagnostic accuracy up to $>90 \%$ and proved to be useful mainly early on in the learning period.

In a large series of patients with solid pancreatic lesions, Iglesias-Garcia et al. [30] observed that the absence of ROSE was associated with a significantly higher rate of inadequate sampling, lower diagnostic sensitivity and overall accuracy for malignancy, and a greater number of passes. The influence of ROSE in determining high diagnostic accuracy of EUS-FNA was confirmed by two meta-analyses including more than 100 studies [31,32]. ROSE remained a significant determinant of EUS-FNA accuracy after correction for study population number and reference standard using metaregression model analysis; [32] the availability of ROSE improved the adequacy rates for EUS-FNA of solid pancreatic lesions by up to $3.5 \%$ [33]. In contrast, the importance of ROSE was not confirmed in a multicenter randomized controlled trial in consecutive patients with solid pancreatic mass undergoing EUS-FNA. No difference in the diagnostic yield of malignancy was demonstrated in two study groups with and without ROSE, although in the latter group seven passes were performed [33]. In our study, the number of passes decreased during the learning period from five at the beginning to three at the end of the study period. No significant differences were observed in terms of diagnostic accuracy.

Of note, in this study EUS-FNA showed a specificity of $100 \%$, even in the early phase, confirming that the technique is an efficient diagnostic tool providing pathological results able to differentiate pancreatic malignancies from benign lesions. As false-negative result of FNA may affect specificity, all patients with negative samples were followed up for at least 1 year to exclude the possibility of misdiagnosis.

Needle size is hypothesized to affect diagnostic accuracy according to the amount of tissue collected for cytological or histological analysis. In general, the standard needle for pancreatic EUS-FNA is a 22-gauge, but choice of needle size is guided by the presumed histological type and location of the target lesion. Ideally, a 19-gauge needle allowing the collection of a larger amount of cellular material compared to a thinner 22-gauge needle should provide a better diagnostic yield and should be preferred in the diagnosis of pancreatic tumors other than pancreatic adenocarcinoma, tumors surrounded by chronic pancreatitis, lymphoma, and autoimmune pancreatitis. However, these advantages may be offset by a higher rate of technical failures in the event of a lesion to biopsy through the second part of the duodenum. In a randomized controlled trial by Song et al. [34], technical failure using 19-gauge needles was reported in 19\% of patients with pancreatic head masses. The main reason which complicates interpretation of the specimen was contamination by blood. A multicenter study showed that a thinner needle provides less cellular material than a larger one, but that the material is more easily interpreted as there is less contamination of the specimen by blood compared to sampling with a larger gauge needle. [35] Minimal differences in diagnostic accuracy between 22-gauge and 25-gauge needles are reported [35-41]. Ultrathin needles could be chosen for pancreatic tumors located in the pancreatic uncinate process because of their superior manageability, and in the case of cytological diagnosis. In the present series, a small number of procedures were carried out using a 19gauge needle, all in the initial study period; it was therefore not possible to evaluate the difference in diagnostic accuracy between 19- and 22-gauge needles. In a small group of patients with pancreatic head lesions, changing the needle from 22- to 25-gauge during the procedure increased diagnostic accuracy in the absence of ROSE. This can be explained by the greater flexibility of thinner needles in the duodenum enabling the collection of tissue from the center of the lesion. We observed a considerable increase in diagnostic accuracy using a new FNB needle, albeit in the final part of the study period. These new needles are able to harvest a core tissue specimen with better preservation of cellular architecture than FNA needles, [42], more representative for the pathologist, and easy to interpret. Moreover, core biopsy needles procured adequate samples useful for ancillary molecular diagnosis. A further increase in diagnostic accuracy was observed using EUS-FNB [42] in the absence of ROSE. A recent study reported that EUS-FNB yielded higherquality specimens for histological diagnosis and better discrimination between pancreatic adenocarcinoma and nonadenocarcinoma tumor [43]. The major advantage of FNB over FNA needles seems to be in the assessment of pancreatic neuroendocrine tumors before surgical resection, as they provide a greater quantity of material for Ki-67 analysis, and in the evaluation of tumor grade [44]. A comparison between needles in terms of specimen adequacy or complications was not performed in the present study due to the low number of FNB performed.

The study has some limitations. First, it is a retrospective investigation, even if data regarding the procedure and patient characteristics were prospectively recorded. The number of interventional procedures per year, especially at the beginning of the period considered, was limited and this could have contributed to extending the length of the training period. EUS-FNAs were carried out by one endosonographer, who began interventional EUS without supervised training and without the assistance of an expert pathologist in bilio-pancreatic disease. Second since most of the sampling was collected using a 22-gauge needle, there are insufficient data to quantify the influence of needle size on the diagnostic accuracy of EUS-FNA. Nevertheless, the present study provides useful insight into the learning process based on the real-life day-to-day experience of an endosonographer performing EUS in the absence of an on-site evaluation of tissue sample adequacy.

In conclusion, this study confirms that EUS-FNA and EUSFNB are safe and efficient procedures for collecting tissue 
from pancreatic mass and biliary tract with good diagnostic accuracy. Operator expertise is a crucial factor influencing the diagnostic accuracy of interventional EUS, particularly in the absence of ROSE. Whether the use of EUS-FNB is able to reduce both the time required to reach a diagnostic accuracy $>85 \%$ and the number of passes needed to obtain sufficient tissue during the learning period could be the subject of a further study.

Conflict of interest: The authors have no conflicts of interest or financial ties to disclosure.

\section{References}

[1] Chang KJ, Nguyen PM, Erickson RA, et al. The clinical utility of endoscopic ultrasound-guided fine-needle aspiration in the diagnosis and staging of pancreatic carcinoma. Gastrointest Endosc. 1997;45(5):387-393.

[2] Hewitt MJ, McPhail MJ, Possamai L, et al. EUS-guided FNA for diagnosis of solid pancreatic neoplasms: a meta-analysis. Gastrointest Endosc. 2012;75(2):319-331.

[3] Wiersema MJ, Vilmann P, Giovannini M, et al. Endosonographguided fine-needle aspiration biopsy: diagnostic accuracy and complication assessment. Gastroenterology. 1997;112(4): 1087-1095.

[4] Dumonceau JM, Polkowski M, Larghi A, European Society of Gastrointestinal Endoscopy, et al. Indications, results, and clinical impact of endoscopic ultrasound (EUS)-guided sampling in gastroenterology: European Society of Gastrointestinal Endoscopy (ESGE) Clinical Guideline. Endoscopy. 2011;43(10):897-912.

[5] Del Vecchio Blanco G, Coppola M, Mannisi E, et al. Impact of endoscopic ultrasound-guided fine-needle aspiration and multidisciplinary approach in the management of abdominal or mediastinal mass. Eur J Gastroenterol Hepatol. 2015;27(9):1045-1051.

[6] Dumonceau JM, Deprez PH, Jenssen C, et al. Indications, results, and clinical impact of endoscopic ultrasound (EUS)-guided sampling in gastroenterology: European Society of Gastrointestinal Endoscopy (ESGE) Clinical Guideline - Updated January 2017. Endoscopy. 2017;49(7):695-714.

[7] Anand D, Barroeta JE, Gupta PK, et al. Endoscopic ultrasound guided fine needle aspiration of non-pancreatic lesions: an institutional experience. J Clin Pathol. 2007;60(11):1254-1262.

[8] Srinivasan R, Bhutani MS, Thosani N, et al. Clinical impact of EUSFNA of mediastinal lymph nodes in patients with known or suspected lung cancer or mediastinal lymph nodes of unknown etiology. J Gastrointes Liver Dis. 2012;21:145-152.

[9] Buscarini E, De Angelis C, Arcidiacono PG, et al. Multicentre retrospective study on endoscopic ultrasound complications. Dig Liver Dis. 2006;38(10):762-767.

[10] Adler DG, Jacobson BC, Davila RE, ASGE, et al. ASGE guideline: complications of EUS. Gastrointest Endosc. 2005;61(1):8-12.

[11] van Riet PA, Larghi A, Attili F, et al. A multicenter randomized trial comparing a 25-gauge EUS fine-needle aspiration device with a 20-gauge EUS fine-needle biopsy device. Gastrointest Endosc. 2019;89(2):329-339.

[12] Nakai $Y$, Isayama $H$, Chang $\mathrm{KJ}$, et al. Slow pull versus suction in endoscopic ultrasound-guided fine- needle aspiration of pancreatic solid masses. Dig Dis Sci. 2014;59(7):1578-1585.

[13] Saxena P, El Zein M, Stevens T, et al. Stylet slow-pull versus standard suction for endoscopic ultrasound-guided fine-needle aspiration of solid pancreatic lesions: a multicenter randomized trial. Endoscopy. 2018;50(5):497-504.

[14] Villa NA, Berzosa M, Wallace MB, et al. Endoscopic ultrasoundguided fine needle aspiration: The wet suction technique. Endosc Ultrasound. 2016;5(1):17-20.

[15] Hoffman BJ, B.Wallace M, Eloubeidi MA, et al. How many supervised procedures does it take to become competent in EUS?
Results of a multicentre three-year study. Gastrointest Endosc. 2000;51(4):AB139.

[16] Mertz H, Gautam S. The learning curve for EUS-guided FNA of pancreatic cancer. Gastrointest Endosc. 2004;59(1):33-37.

[17] Harewood GC, Wiersema LM, Halling AC, et al. Influence of EUS training and pathology interpretation on accuracy of EUS-guided fine needle aspiration of pancreatic masses. Gastrointest Endosc. 2002;55(6):669-673.

[18] Eisen GM, Dominitz JA, Faigel DO, American Society for Gastrointestinal Endoscopy, et al. Guidelines for credentialing and granting privileges for endoscopic ultrasound. Gastrointest Endosc. 2001;54(6):811-814.

[19] Eloubeidi MA, Tamhane A. EUS-guided FNA of solid pancreatic masses: a learning curve with 300 consecutive procedures. Gastrointest Endosc. 2005;61(6):700-708.

[20] American Society for Gastrointestinal Endoscopy. Role of EUS. Gastrointest Endosc. 2000;52:852-859.

[21] Polkowski M, Larghi A, Weynand B, European Society of Gastrointestinal Endoscopy (ESGE), et al. Learning, techniques, and complications of endoscopic ultrasound (EUS)-guided sampling in gastroenterology: European Society of Gastrointestinal Endoscopy (ESGE) Technical Guideline. Endoscopy. 2012;44(2): 190-206.

[22] Khoury T, Kadah A, Farraj M, et al. The role of rapid on-site evaluation on diagnostic accuracy of endoscopic ultrasound fine needle aspiration for pancreatic, submucosal upper gastrointestinal tract and adjacent lesions. Cytopathology. 2019;30(5):499-503.

[23] Fisher L, Segarajasingam DS, Stewart C, et al. Endoscopic ultrasound guided fine needle aspiration of solid pancreatic lesions: performance and outcomes. J Gastroenterol Hepatol. 2009;24(1): 90-96.

[24] Polkowski M, Jenssen C, Kaye $P$, et al. Technical aspects of endoscopic ultrasound (EUS)-guided sampling in gastroenterology: European Society of Gastrointestinal Endoscopy (ESGE) Technical Guideline - March 2017. Endoscopy. 2017;49(10):989-1006.

[25] DiMaio CJ, Mishra G, McHenry L, ASGE Training Committee, et al. EUS core curriculum. Gastrointest Endosc. 2012;76(3):476-481.

[26] Wani S, Wallace MB, Cohen J, et al. Quality indicators for EUS. Gastrointest Endosc. 2015;81(1):67-80.

[27] Azad JS, Verma D, Kapadia AS, et al. Can U.S. GI fellowship programs meet American Society for Gastrointestinal Endoscopy recommendations for training in EUS? A survey of U.S. GI fellowship program directors. Gastrointest Endosc. 2006;64(2):235-241.

[28] Wani S, Coté GA, Keswani R, et al. Learning curves for EUS by using cumulative sum analysis: implications for American Society for Gastrointestinal Endoscopy recommendations for training. Gastrointest Endosc. 2013;77(4):558-565.

[29] Kim J, Ryu JK, Park JM, et al. Clinical factors associated with accuracy of EUS-FNA for pancreatic or peripancreatic solid mass without on-site cytopathologists. J Gastroenterol Hepatol. 2014; 29(4):887-892.

[30] Iglesias-Garcia J, Dominguez-Munoz JE, Abdulkader I, et al. Influence of on-site cytopathology evaluation on the diagnostic accuracy of endoscopic ultrasound-guided fine needle aspiration (EUS-FNA) of solid pancreatic masses. Am J Gastroenterol. 2011; 106:1705.

[31] Hébert-Magee S, Bae S, Varadarajulu S, et al. The presence of a cytopathologist increases the diagnostic accuracy of endoscopic ultrasound-guided fine needle aspiration cytology for pancreatic adenocarcinoma: a meta-analysis. Cytopathology. 2013;24(3): 159-171.

[32] Matynia AP, Schmidt RL, Barraza G, et al. Impact of rapid on-site evaluation on the adequacy of endoscopic-ultrasound guided fine-needle aspiration of solid pancreatic lesions: a systematic review and meta-analysis. J Gastroenterol Hepatol. 2014;29(4): 697-705.

[33] Wani S, Mullady D, Early DS, et al. The clinical impact of immediate onsite cytopathology evaluation during endoscopic ultrasound-guided fine needle aspiration of pancreatic masses: a 
prospective multicenter randomized controlled trial. Am J Gastroenterol. 2015;110:1429-1439.

[34] Song TJ, Kim JH, Lee SS, et al. The prospective randomized, controlled trial of endoscopic ultrasound-guided fine-needle aspiration using $22 \mathrm{G}$ and $19 \mathrm{G}$ aspiration needles for solid pancreatic or peripancreatic masses. Am J Gastroenterol. 2010;105(8): 1739-1745.

[35] Yusuf TE, Ho S, Pavey DA, et al. Retrospective analysis of the utility of endoscopic ultrasound-guided fine-needle aspiration (EUSFNA) in pancreatic masses, using a 22-gauge or 25-gauge needle system: a multicenter experience. Endoscopy. 2009;41(5):445-448.

[36] Affolter KE, Schmidt RL, Matynia AP, et al. Needle size has only a limited effect on outcomes in EUS-guided fine needle aspiration: a systematic review and meta-analysis. Dig Dis Sci. 2013;58(4): 1026-1034.

[37] Camellini L, Carlinfante G, Azzolini F, et al. A randomized clinical trial comparing $22 \mathrm{G}$ and $25 \mathrm{G}$ needles in endoscopic ultrasoundguided fine-needle aspiration of solid lesions. Endoscopy. 2011; 43(8):709-715.

[38] Fabbri C, Polifemo AM, Luigiano C, et al. Endoscopic ultrasoundguided fine needle aspiration with 22- and 25-gauge needles in solid pancreatic masses: a prospective comparative study with randomisation of needle sequence. Dig Liver Dis. 2011;43(8): 647-652.
[39] Lee JK, Lee KT, Choi ER, et al. A prospective, randomized trial comparing 25-gauge and 22-gauge needles for endoscopic ultrasound-guided fine needle aspiration of pancreatic masses. Scand J Gastroenterol. 2013;48(6):752-757.

[40] Madhoun MF, Wani SB, Rastogi A, et al. The diagnostic accuracy of 22-gauge and 25-gauge needles in endoscopic ultrasoundguided fine needle aspiration of solid pancreatic lesions: a metaanalysis. Endoscopy. 2013;45(2):86-92.

[41] Varadarajulu S, Hasan MK, Bang JY, et al. Endoscopic ultrasound-guided tissue acquisition. Dig Endosc. 2014;26(Suppl 1): 62-69.

[42] Di Mitri RD, Rimbas M, Attili F, et al. Performance of a new needle for endoscopic ultrasound-guided fine-needle biopsy in patients with pancreatic solid lesions: a retrospective multicenter study. Endosc Ultrasound. 2018;7(5):329-334.

[43] Jiang H, Guo J, Wang K, et al. 22-Gauge biopsy needles have a better histological diagnostic yield in the discrimination of specific pancreatic solid neoplasms. Scand J Gastroenterol. 2019; 54(1):101-107.

[44] Leeds JS, Nayar MK, Bekkali NLH, et al. Endoscopic ultrasoundguided fine-needle biopsy is superior to fine-needle aspiration in assessing pancreatic neuroendocrine tumors. Endosc Int Open. 2019;07(10):E1281-E1287. 